\title{
Force, relative-displacement, and work networks in granular materials subjected to quasistatic deformation
}

\author{
N. P. Kruyt* \\ Department of Mechanical Engineering, University of Twente, P.O. Box 217, 7500 AE Enschede, The Netherlands \\ S. J. Antony \\ Institute of Particle Science and Engineering, School of Process, Environmental and Materials Engineering, University of Leeds, \\ Leeds LS2 9JT, United Kingdom
}

(Received 20 October 2006; published 24 May 2007)

\begin{abstract}
To describe the heterogeneous nature of stress transmission in granular materials, the concept of the "strong" network consisting of contacts with large normal forces has been proposed by Radjaï et al. [Phys. Rev. Lett. 80, 61 (1998)]. The shear stress is mainly determined by this strong network. The dual viewpoint is adopted here, by not only considering the forces at contacts, but also the deformation. It is shown that the strain increments are determined by the tangential component of the relative displacements at the contacts. A "mobile" network consisting of contacts with large tangential relative displacements is defined that primarily accounts for the strain increments. The investigation of the relation between the strong and the mobile networks shows that these networks are largely unrelated. An alternative network is defined that consists of contacts at which the contribution to the work input is large. It is found that this work input occurs primarily through the tangential forces and tangential relative displacements.
\end{abstract}

DOI: 10.1103/PhysRevE.75.051308

PACS number(s): 81.05.Rm, 83.80.Fg

\section{INTRODUCTION}

Micromechanics of quasistatic deformation of granular materials deals with the relation between characteristics at the microscopic level and those at the macroscopic, continuum level. Important objectives are to increase the understanding of the mechanical behavior of quasistatic deformation of granular materials, as well as (more ambitiously) to develop micromechanically based constitutive relations. Valuable methods for the former objective are the Discrete Element Method (DEM), a computer simulation method originally developed by Cundall and Strack [1], and photoelastic experiments (for example, [2-5]). From DEM simulations and from photoelastic experiments, force chains were observed: these are chainlike structures of (relatively) high forces, which are correlated over a length scale that is (much) larger than the average particle size. Thus the force transmission (and hence the stress distribution) in granular materials is very heterogeneous. This led to the concept of the "strong" network [6], based on the analysis of results of DEM simulations. The strong network is defined as the set of contacts at which the normal force is "large." It was shown by Radjaï et al. [6] that the shear stress is mainly determined by the strong network, while the strong and the "weak" networks both contribute to the mean pressure.

A procedure to objectively determine the force chains is given by Peters et al. [7]. A continuum description for the stress tensor that takes into account the concept of the strong network has been formulated by Cates et al. [8,9].

For a proper understanding of the mechanical behavior of granular materials, knowledge of the contact forces is not

\footnotetext{
*Electronic address: n.p.kruyt@utwente.nl

†Electronic address: s.j.antony@leeds.ac.uk
}

sufficient, since these forces are linked to the deformation. Therefore, the present investigation extends the study by Radjaï et al. [6] by also considering the dual viewpoint. Hence the deformation at contacts, i.e., the relative displacements between particles that are in contact, is also considered. Previous studies have shown that the deformation is very heterogeneous [2,10-12], even when no shear band has formed. The deformation occurs at "microbands" that separate "blocks" of particles. Significant deformation occurs at only a fraction of the total number of contacts.

Based on these observations, a division of the set of contacts is proposed here that is similar to the division in the strong and weak contacts, namely the division in "mobile" and "immobile" contacts. At mobile contacts there is significant deformation, while at immobile contacts there is only small deformation. The mobile network then consists of the mobile contacts. An alternative network, the "active" network, based on consideration of the work input, is also examined. The method used here to investigate the force and relative-displacement networks is that of two-dimensional DEM simulation.

The outline of this study is as follows. In Sec. II the basics of micromechanics of quasistatic deformation of granular materials are recapitulated. In Sec. III the performed DEM simulation is described. Force and relative-displacement networks are investigated in Secs. IV and V, respectively. The relations between these force and relative-displacement networks are studied in Sec. VI. The network based on workinput considerations is explored in Sec. VII. Finally, findings from this study are discussed.

\section{MICROMECHANICS}

At the microscopic level of stiff, interacting particles the important quantities are the contact force $f_{i}^{r s}$, i.e., the force 
exerted on particle $r$ by particle $s$, and the contact relativedisplacement increment $d \Delta_{i}^{r s}$ between the particles $r$ and $s$. The contact relative-displacement increment $d \Delta_{i}^{r s}$, based on the relative displacements of the particles centers, is defined by $d \Delta_{i}^{r s}=d U_{i}^{s}-d U_{i}^{r}$, where $d U_{i}^{r}$ is the increment of the displacement of the center of particle $r$.

At the macroscopic, continuum level the important quantities in quasistatic deformation are the average stress tensor $\sigma_{i j}$ and the average strain-increment tensor $d \epsilon_{i j}$. The macroscopic stress tensor and the strain-increment tensor are related to the microscopic contact force and contact relative displacement by suitable averages. These are the micromechanical expressions for stress and strain.

The micromechanical expression for the average stress tensor $\sigma_{i j}$ adopted here is, for the two-dimensional case considered (see, for example, $[2,13,14]$ )

$$
\sigma_{i j}=\frac{1}{S} \sum_{c \in C} f_{i}^{c} l_{j}^{c}
$$

where $C$ is the set of contacts in the region of interest with area $S, f_{i}^{c}$ is the force at contact $c$, and $l_{i}^{c}$ is the branch vector at contact $c$. The branch vector $l_{i}^{c}$ is defined as the vector that connects the centers of particles that are in contact.

The micromechanical expression for the average strainincrement tensor is, for the two-dimensional case, considered [14],

$$
d \epsilon_{i j}=\frac{1}{S} \sum_{c \in C} d \Delta_{i}^{c} h_{j}^{c},
$$

where $d \Delta_{i}^{c}$ is the increment of the relative displacement at contact $c$ and $h_{i}^{c}$ is the polygon vector at contact $c$. The polygon vector is obtained from the rotated polygon vector $g_{i}^{c}$ by counterclockwise rotation over $90^{\circ}$. The rotated polygon vector is defined as the vector that connects the centers of adjacent polygons. These polygons arise as a way of partitioning the plane network of particle centers and contacts $[14,15]$.

The micromechanical expression for the stress tensor, Eq. (1), seems to be well-established, although some controversies remain [16-18] and new approaches have recently been proposed $[15,19,20]$. The micromechanical expression for the strain-increment tensor [Eq. (2)] does not involve particle rotations. The proper way of accounting for particle rotations in measures for macroscopic deformation does not seem to be fully resolved $[15,21,22]$.

The contact force vector $f_{i}^{c}$ can be decomposed into normal and tangential components $f_{n}^{c}$ and $f_{t}^{c}$, such that $f_{i}^{c}=f_{n}^{c} n_{i}^{c}$ $+f_{t}^{c} t_{i}^{c}$, where $n_{i}^{c}$ and $t_{i}^{c}$ are the unit normal and tangential vector at the contact. Similarly, the normal and tangential components of the relative-displacement increment are denoted by $d \Delta_{n}^{c}$ and $d \Delta_{t}^{c}$, respectively.

The stress and strain-increment tensors can be split into various contributions. The contributions that will be considered here are (i) the contributions due to normal and tangential force or relative displacement and (ii) the contributions due to subsets of contacts.

For future reference, the stress tensor $\sigma_{i j}$ and the strainincrement tensor $d \epsilon_{i j}$ are split into terms $\sigma_{i j}^{N}, \sigma_{i j}^{T}$ and $d \epsilon_{i j}^{N}$, $d \epsilon_{i j}^{T}$, corresponding to the contributions of the normal and tangential components of the force and relative-displacement increments at contacts, respectively,

$$
\begin{aligned}
& \sigma_{i j}^{N}=\frac{1}{S} \sum_{c \in C}\left[f_{n}^{c} n_{i}^{c}\right] l_{j}^{c}, \quad \sigma_{i j}^{T}=\frac{1}{S} \sum_{c \in C}\left[f_{t}^{c} t_{i}^{c}\right] l_{j}^{c}, \\
& d \epsilon_{i j}^{N}=\frac{1}{S} \sum_{c \in C}\left[d \Delta_{n}^{c} n_{i}^{c}\right] h_{j}^{c}, \quad d \epsilon_{i j}^{T}=\frac{1}{S} \sum_{c \in C}\left[d \Delta_{t}^{c} t_{i}^{c}\right] h_{j}^{c} .
\end{aligned}
$$

Note that $\sigma_{i j}=\sigma_{i j}^{N}+\sigma_{i j}^{T}$ and $d \epsilon_{i j}=d \epsilon_{i j}^{N}+d \epsilon_{i j}^{T}$.

The partial stress tensor $\sigma_{i j}\left(C_{\text {sub }}\right)$ and partial strainincrement tensor $d \epsilon_{i j}\left(C_{\text {sub }}\right)$ corresponding to a subset $C_{\text {sub }}$ of the total set of contacts $C$ are defined by [compare the micromechanical expressions for the stress and strain tensors, Eqs. (1) and (2), respectively]

$$
\sigma_{i j}\left(C_{\mathrm{sub}}\right)=\frac{1}{S} \sum_{c \in C_{\mathrm{sub}}} f_{i}^{c} l_{j}^{c}, \quad d \epsilon_{i j}\left(C_{\mathrm{sub}}\right)=\frac{1}{S} \sum_{c \in C_{\mathrm{sub}}} d \Delta_{i}^{c} h_{j}^{c} .
$$

Note that the (total) stress tensor $\sigma_{i j}$ and the (total) strainincrement tensor $d \epsilon_{i j}$ are retrieved when $C_{\text {sub }}=C$.

\section{DEM SIMULATION}

The contact constitutive relation, which relates the force and the relative displacement at the contact level, used here in the DEM simulation is that employed in [1]. This contact constitutive relation involves elastic and Coulomb frictional effects. The elastic component of the constitutive relation is described by two linear springs in the normal and tangential direction at the contact with spring constants $k_{n}$ and $k_{t}$, respectively. Only compressive normal forces are allowed. If the normal force were to become negative, the contact is considered to be disrupted for cohesionless materials. Furthermore, the tangential force is limited by Coulomb friction, i.e., $\left|f_{t}^{c}\right| \leqslant \mu f_{n}^{c}$, where $\mu$ is the interparticle friction coefficient.

The two-dimensional simulation of biaxial deformation (in which the principal strain $\epsilon_{1}$ is imposed and the principal stress $\sigma_{2}$ is kept constant) has been performed for an assembly consisting of 50000 disks from a lognormal particle-size distribution for which the ratio of the standard deviation over the mean equals 0.25 . An initial assembly has been created in a separate DEM simulation by starting from a sparse state and then isotropically compressing until the desired isotropic pressure was obtained. The microstructure of this initial assembly is isotropic. The packing fraction $\rho$, i.e., the area occupied by the particles divided by the total area occupied by the assembly, of the initial assembly is $\rho=0.843$. The corresponding coordination number, i.e., the average number of contacts per particle, is $\Gamma=4.17$. The confining stress in this dense initial isotropic state is denoted by $\sigma_{0}$. The parameters in the contact constitutive relation are selected such that $k_{t} / k_{n}=0.5, \mu=0.5$, and $\sigma_{0} / k_{n}=10^{-4}$, where the latter quantity is nondimensional in the two-dimensional case considered here. The ratio $\sigma_{0} / k_{n}$ is small, so particle deformation is small and the response is stiff in the elastic regime. Periodic 


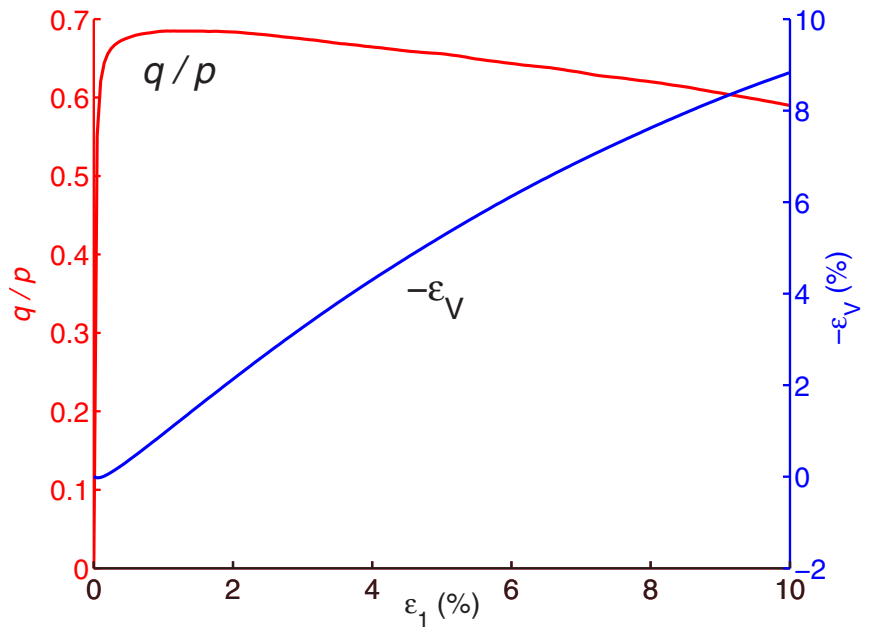

FIG. 1. (Color online) Macroscopic response: evolution with imposed strain $\epsilon_{1}$ of shear strength $q / p$ and volumetric strain $\epsilon_{V}$.

boundary conditions have been employed to minimize boundary effects and to suppress the formation of shear bands in order to also investigate the behavior at larger strains.

Invariants of the stress tensor $\sigma_{i j}$ are the mean pressure $p=1 / 2\left(\sigma_{1}+\sigma_{2}\right)$ and the shear stress $q=1 / 2\left(\sigma_{1}-\sigma_{2}\right)$, with principal stresses $\sigma_{1}$ and $\sigma_{2}$. Invariants of the strain tensor $\epsilon_{i j}$ are the volumetric strain $\epsilon_{V}=\epsilon_{1}+\epsilon_{2}$ and the shear strain $\epsilon_{S}=\epsilon_{1}-\epsilon_{2}$, with principal strains $\epsilon_{1}$ and $\epsilon_{2}$. The macroscopic response, in terms of the evolution with imposed strain $\epsilon_{1}$ of shear strength $q / p$ and of volumetric strain $\epsilon_{V}$, is shown in Fig. 1. The observed macroscopic behavior is typical for a dense initial assembly. Note that this dense system, which includes frictional effects, is far removed from a jammingunjamming transition, as studied in [23-26] for frictionless systems.

\section{FORCE NETWORK}

The description of the force network, as proposed in [6], is recapitulated to emphasize the duality with the relativedisplacement network defined in Sec. V. Furthermore, results on the force network at large strains are given.

The relative contributions of the normal and tangential contact forces to the shear stress, in both two- and threedimensional DEM simulations, have been determined in [27-29]. They noted that the stress is mainly contributed by the normal component of the contact forces. This was demonstrated analytically in [30].

The mean pressure and shear stress corresponding to $\sigma_{i j}^{N}$, as defined in Eq. (3), are denoted by $p^{N}$ and $q^{N}$, respectively. It can be shown that for assemblies of two-dimensional disks $p^{N}=p$, i.e., the tangential forces do not contribute to the mean pressure: $p^{T}=0$. For $\epsilon_{1}>0.5 \%$, we find from the DEM simulation that $q^{N} / q \cong 0.86$. Thus when considering the characteristics of the contact network with respect to force transmission, it suffices to focus on the normal forces, consistent with the findings of [27-29].

The set of contacts $C_{f}(\alpha)$ (where $0 \leqslant \alpha \leqslant 1$ is a nondimensional parameter) is defined such that $P_{f}\left(f_{n}^{c}\right) \leqslant \alpha$ for all con-
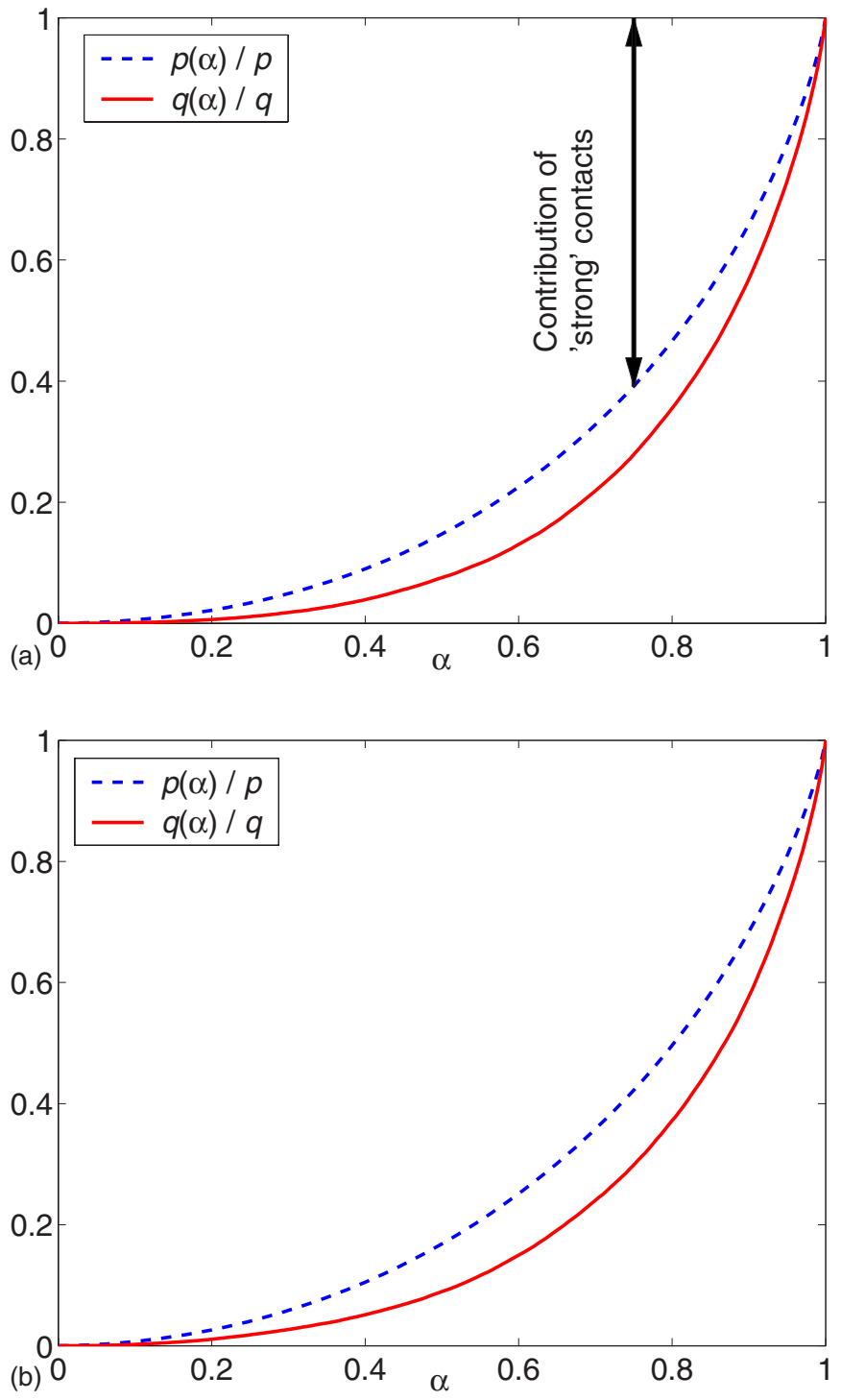

FIG. 2. (Color online) Partial pressure $p(\alpha)$ and partial shear stress $q(\alpha)$ at peak shear strength (top) and at large strain (bottom).

tacts $c \in C_{f}(\alpha)$. Here $P_{f}\left(f_{n}\right)$ is the cumulative probability density function for the normal forces. Thus if $c \in C_{f}(\alpha)$, its normal force is smaller than the $\alpha$-quantile. The strong network consists of the contacts in the complement of $C_{f}(\alpha)$, i.e., $C-C_{f}(\alpha)$. Probability density functions for the normal and tangential components of the forces at contacts have been studied in $[5,31,32]$.

The partial stress tensor $\sigma_{i j}(\alpha)$ is a concise notation for the partial stress corresponding to the set $C_{f}(\alpha)$, see also the definition of the partial stress in Eq. (5). The corresponding partial mean pressure and shear stress are denoted by $p(\alpha)$ and $q(\alpha)$; note that $p(\alpha=1)=p$ and $q(\alpha=1)=q$. The partial mean pressure and partial shear stress are shown in Fig. 2 at peak shear strength and at large strain for various values of $\alpha$. We observe from Fig. 2 that at peak shear strength for $\alpha=0.5, p(\alpha) / p=0.15$ and $q(\alpha) / q=0.08$. This means that the $50 \%$ of the contacts at which the normal force is smaller than the median contact normal force, contribute $15 \%$ to the total pressure and only $8 \%$ to the shear stress. Since for $\alpha=0.9$, 
$p(\alpha) / p=0.66$ and $q(\alpha) / q=0.57$, the strongest $10 \%$ of the contacts contribute $34 \%$ to the total pressure and $43 \%$ to the total shear stress. Thus the strong contacts mainly determine the shear stress and, to a lesser extent, the mean pressure. The behavior at large strain is similar to that at peak shear strength, see Fig. 2. The influence of particle shape on these stress quantities has been studied in [33].

\section{RELATIVE-DISPLACEMENT NETWORK}

In Sec. IV the contributions $\sigma_{i j}^{N}$ and $\sigma_{i j}^{T}$ of the normal and of the tangential forces to the stress tensor $\sigma_{i j}$ have been considered. It has been shown that the contribution of the normal forces is dominant.

In this section the contributions $d \epsilon_{i j}^{N}$ and $d \epsilon_{i j}^{T}$, as defined in Eq. (4), of the normal and tangential components of the relative-displacement increments at contacts to the strainincrement tensor $d \epsilon_{i j}$ are considered. The corresponding volumetric and shear strain increments are $d \epsilon_{V}^{N}$ and $d \epsilon_{S}^{N}$ for the normal relative displacements, while $d \epsilon_{V}^{T}$ and $d \epsilon_{S}^{T}$ correspond to the tangential component.

The evolution of these quantities with imposed strain $\epsilon_{1}$ is shown in Fig. 3. For $\epsilon_{1}>0.5 \%$ the tangential component of the relative displacement increments practically determines the volumetric strain increment and completely determines the shear strain increment. Thus when considering the characteristics of the contact network with respect to deformation, it suffices to focus on the tangential relative displacements. This means that mean-field assumptions, as often employed in micromechanical studies, are generally incorrect (see also [36-38]).

In analogy to the definition of the set $C_{f}(\alpha)$, the set of contacts $C_{\Delta}(\beta)$ (where $0 \leqslant \beta \leqslant 1$ is a nondimensional parameter) is defined such that $P_{\Delta}\left(\left|d \Delta_{t}^{c}\right|\right) \leqslant \beta$ for all contacts $c$ $\in C_{\Delta}(\beta)$. Here $P_{\Delta}\left(d \Delta_{t}\right)$ is the cumulative probability density function for the absolute values of the increment of the tangential relative displacement. Note that the tangential component of the increment of relative displacement can take either sign and its average is zero. Thus if $c \in C_{\Delta}(\beta)$, its tangential relative displacement is smaller than the $\beta$-quantile. The mobile network consists of the contacts in the complement of $C_{\Delta}(\beta)$, i.e., $C-C_{\Delta}(\beta)$. The contacts that are part of the mobile network are called mobile, while the contacts that are not part of the mobile network are called immobile. Probability density functions of the normal and tangential components of the relative displacements at contacts have been studied in [32]. Those for displacement fluctuations are studied in [34] for granular materials and in [35] for foams.

The partial strain-increment tensor $d \epsilon_{i j}(\beta)$ is a concise notation for the partial strain increment corresponding to the set $C_{\Delta}(\beta)$, see also the definition of the partial strainincrement tensor in Eq. (5). The corresponding volumetric and shear strain increments are shown in Fig. 4 at peak shear strength and at large strain. We observe from Fig. 4 that at peak shear strength for $\beta=0.5, d \epsilon_{V}(\beta) / d \epsilon_{V}=0.11$ and $d \epsilon_{S}(\beta) / d \epsilon_{S}=0.11$. This means that the $50 \%$ of the contacts at which the tangential relative displacement is smaller than the
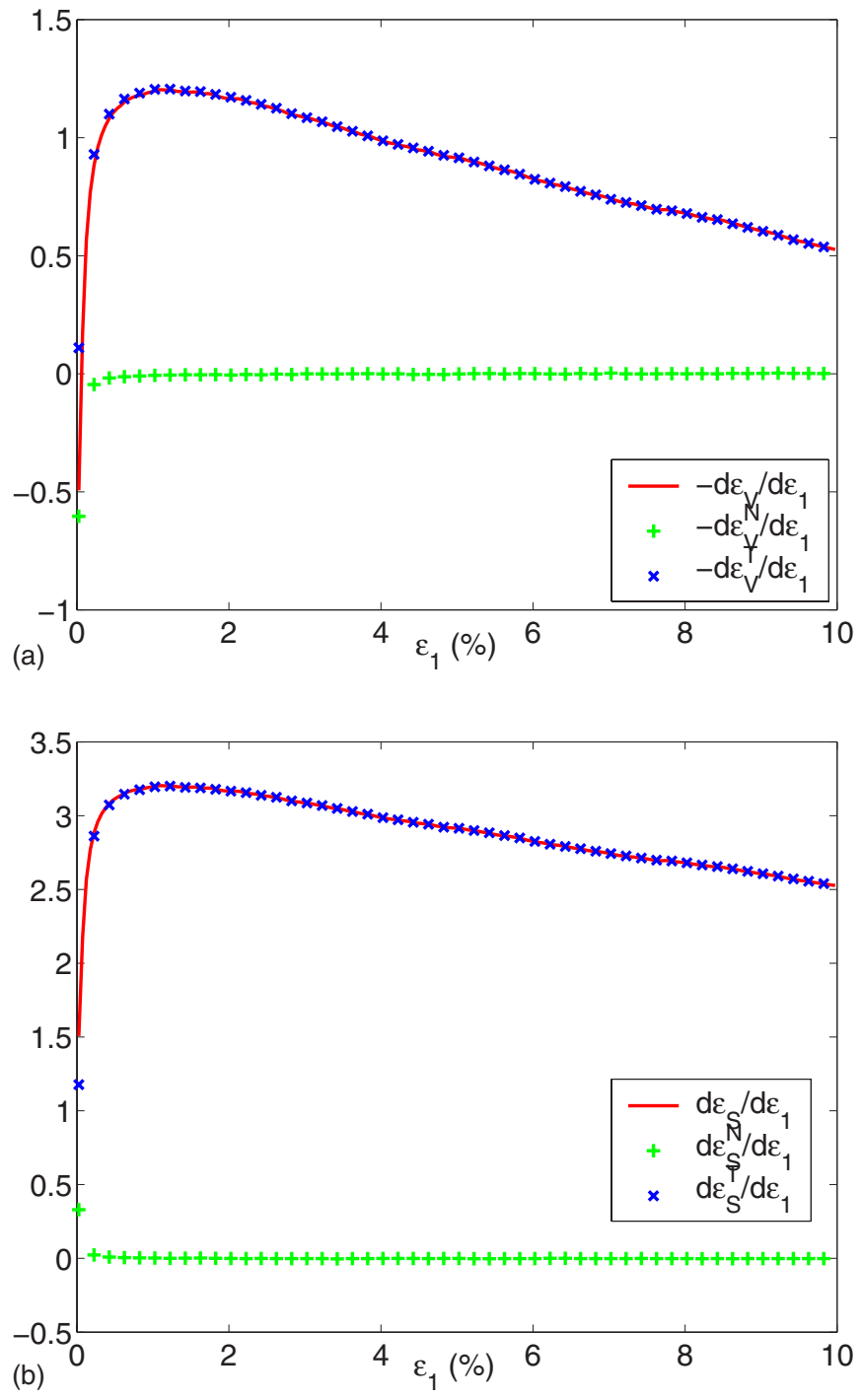

FIG. 3. (Color online) Evolution with imposed strain $\epsilon_{1}$ of contributions due to normal and tangential relative displacements to volumetric strain increment (top) and shear strain increment (bottom).

median tangential relative displacement contribute $11 \%$ to the volumetric strain increment and $11 \%$ to the shear strain increment. Since at peak shear strength for $\beta=0.9$, $d \epsilon_{V}(\beta) / d \epsilon_{V}=0.66$ and $d \epsilon_{S}(\beta) / d \epsilon_{S}=0.66$, the most mobile $10 \%$ of the contacts contribute $34 \%$ to the total volumetric strain increment and 34\% to the total shear strain increment. Thus the mobile contacts determine the volumetric and the shear strain increment. The behavior at large strain is qualitatively similar to that at peak shear strength.

\section{COMPARISON OF STRONG AND MOBILE NETWORKS}

In the previous sections it has been shown that two networks can be identified. The strong network (defined via normal forces) largely accounts for the shear stress and (to a lesser extent) the mean pressure, while the mobile network (defined via tangential relative displacements) largely ac- 

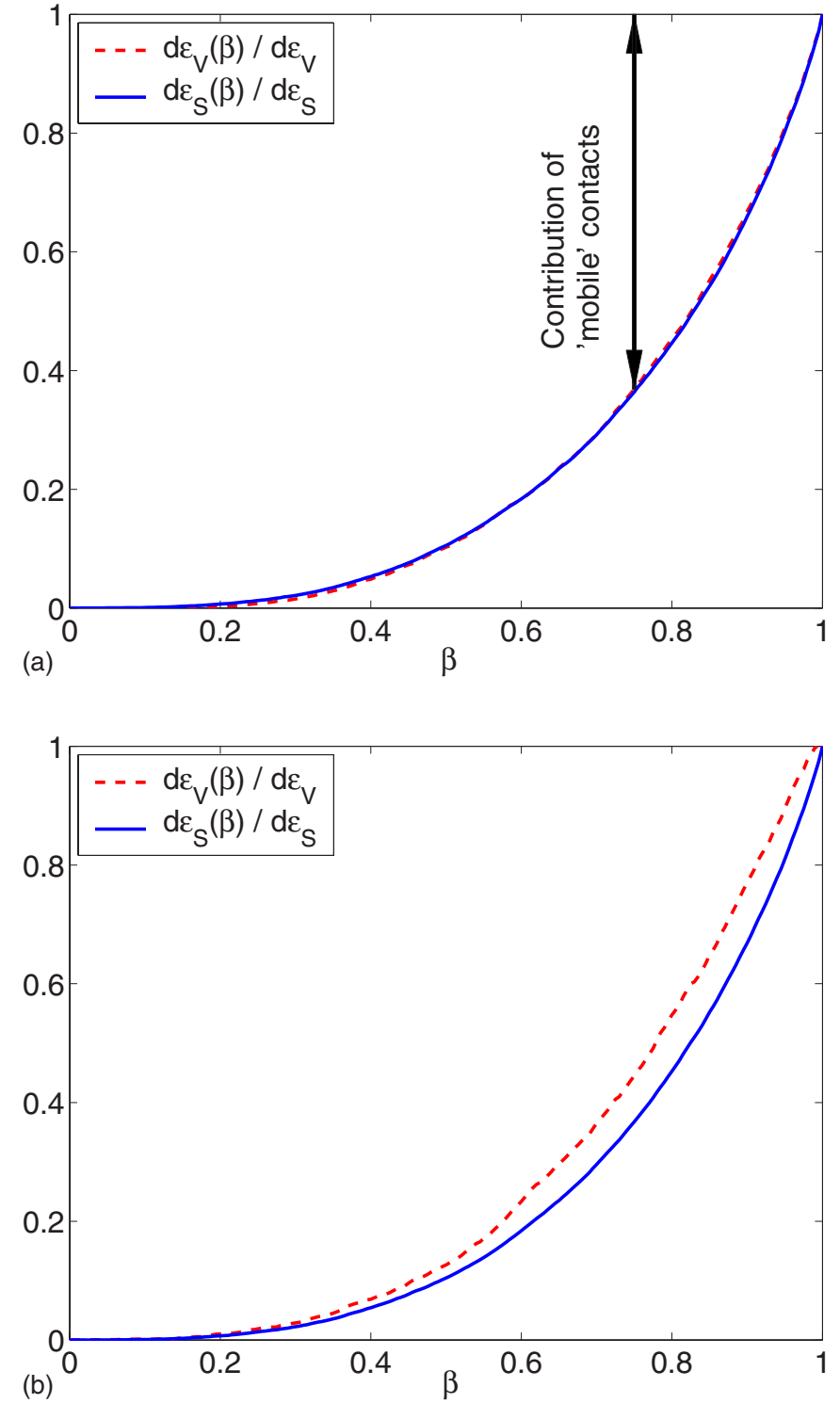

FIG. 4. (Color online) Partial volumetric and strain increment $d \epsilon_{V}(\beta)$ and partial shear strain increment $d \epsilon_{S}(\beta)$ at peak shear strength (top) and at large strain (bottom).

counts for the volumetric and the shear strain increments. Here relations between these networks are investigated.

A visual representation of the two networks is given in Fig. 5. Four groups of contacts are distinguished: strong and mobile, strong and immobile, weak and mobile, and weak and immobile. The force chains are visible, as well as groups of immobile contacts. This visual representation suggests that the two networks are, to a large extent, unrelated (or independent).

This impression is further investigated by considering the percentage of contacts in the four groups. For the specific choice of $\alpha=0.9$ and $\beta=0.9$, i.e., $10 \%$ strong and $10 \%$ mobile contacts, these percentages are given in Table I at peak shear strength and at large strain. Note that since $\alpha=0.9$, the total percentage of strong contacts equals $10 \%$ and the total percentage of weak contacts equals $90 \%$. Similarly, since $\beta=0.9$, the total percentage of mobile contacts equals $10 \%$

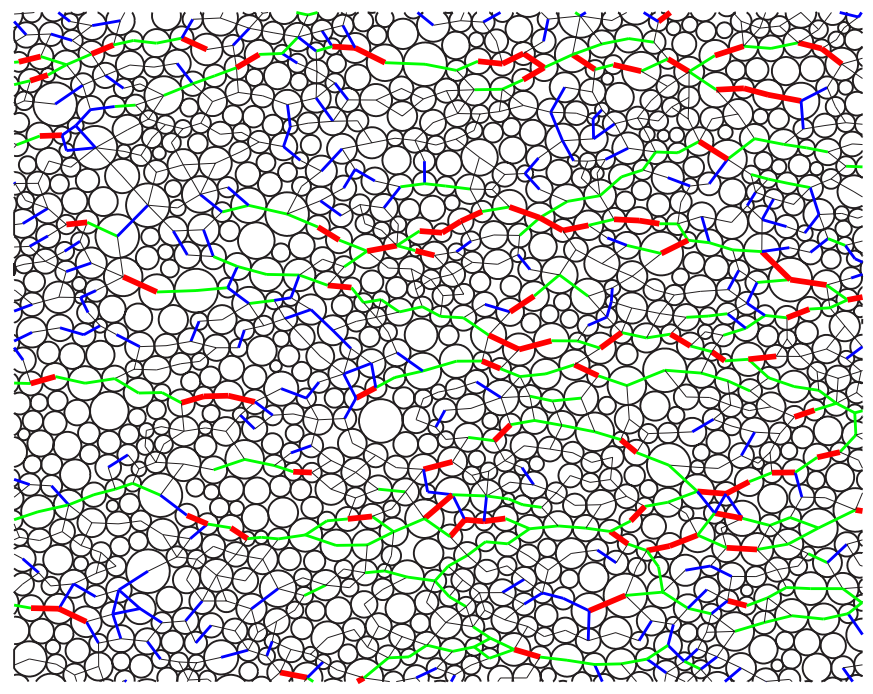

FIG. 5. (Color online) Force and relative-displacement networks for part of the assembly. The color of the contacts signifies the type of contact: red contacts are strong and mobile; green contacts are strong and immobile; blue contacts are weak and mobile; and black contacts are weak and immobile. Result at peak shear strength; $\alpha=\beta=0.8$.

and the total percentage of immobile contacts equals $90 \%$. If the strong and mobile networks were disjoint, then the percentage of contacts that are strong and mobile would equal zero. This is not observed; instead, the results indicate that the two networks are unrelated (or independent): then the percentage of strong and mobile contacts would equal 0.1 $\times 0.1=1 \%$, which is fairly close to the observed value of $1.6 \%$.

The contributions of the strong and the mobile networks to the total mean pressure, shear stress, volumetric, and shear strain increments are shown in Table II. This table shows that the contributions of the $10 \%$ of the mobile contacts (corresponding to $\beta=0.9$ ) to the total mean pressure and shear stress are about $10 \%$ at peak shear strength and at large strain (values range from 0.10 to 0.13 ), indicating that the mobile network is not correlated with the strong network. The contribution of the strong network to the shear strain increment is as expected for uncorrelated networks (values are 0.14 and 0.13 , while for uncorrelated networks the values would equal $0.10)$. The contribution of the strong network to the volumetric strain increment is larger than expected from uncorrelated networks (values are 0.23 and 0.27 , while for uncorrelated

TABLE I. Percentages of various groups of contacts: strong vs mobile network; $\alpha=\beta=0.9$.

\begin{tabular}{lccccc}
\hline \hline & \multicolumn{4}{c}{ Percent of contacts } \\
\cline { 2 - 3 } Type of & \multicolumn{2}{c}{ Peak shear strength } & & \multicolumn{2}{c}{ Large strain } \\
\cline { 2 - 3 } \cline { 5 - 6 } contact & Mobile & Immobile & & Mobile & Immobile \\
\hline Strong & 1.6 & 8.4 & & 1.4 & 8.6 \\
Weak & 8.4 & 81.6 & & 8.6 & 81.4 \\
\hline \hline
\end{tabular}


TABLE II. Stress and strain contributions of various groups of contacts; $\alpha=\beta=0.9$.

\begin{tabular}{lcccccc}
\hline \hline & \multicolumn{2}{c}{ Peak shear strength } & & \multicolumn{2}{c}{ Large strain } \\
\cline { 2 - 3 } \cline { 5 - 6 } Contribution & Strong & Mobile & & Strong & Mobile \\
\hline$p(C) / p$ & 0.34 & 0.13 & & 0.32 & 0.11 \\
$q(C) / q$ & 0.43 & 0.12 & & 0.42 & 0.10 \\
$d \epsilon_{V}(C) / d \epsilon_{V}$ & 0.23 & 0.33 & & 0.27 & 0.23 \\
$d \epsilon_{S}(C) / d \epsilon_{S}$ & 0.14 & 0.34 & & 0.13 & 0.33 \\
\hline \hline
\end{tabular}

networks the values would equal 0.10). A tentative explanation for this observation is as follows. Volumetric strains are mainly induced by tangential relative displacements corresponding to contact reorientations [36]. The larger-thanexpected (relative to uncorrelated networks) volumetric strain increment of the strong network is postulated to be related to such buckling-related reorientations of contacts in the strong network [6].

\section{WORK NETWORK}

The analysis of the force network showed that the major part of the shear stress is due to the strong contacts with large normal forces, while the analysis of the relativedisplacement network showed that the major part of the volumetric and shear strain is due to deformation at the mobile contacts with large tangential relative displacements. In the previous section it has been shown that these two networks are largely unrelated. The question then arises of how the work increment, being a combination of forces and relative-displacement increments, is distributed over the contacts. Total work increment, energy, and dissipation in quasistatic deformation of granular materials have been studied from the macroscopic viewpoint in [39].

The total work increment $\delta W$ is defined as $\delta W=\delta W^{N}$ $+\delta W^{T}$ where the contributions due to the normal and tangential modes, $\delta W^{N}$ and $\delta W^{T}$, respectively, are defined by

$$
\delta W^{N}=\frac{1}{S} \sum_{c \in C} f_{n}^{c} d \Delta_{n}^{c}, \quad \delta W^{T}=\frac{1}{S} \sum_{c \in C} f_{t}^{c} d \Delta_{t}^{c} .
$$

The evolution with imposed strain $\epsilon_{1}$ of the two contributions $\delta W^{N}$ and $\delta W^{T}$ is shown in Fig. 6 in nondimensional form. Beyond the elastic range $\delta W^{N} \ll \delta W^{T}$, so most of the work input occurs through the tangential mode.

In analogy to the definition of the sets $C_{f}(\alpha)$ and $C_{\Delta}(\beta)$, the set of contacts $C_{\delta W}(\gamma)$ (where $0 \leqslant \gamma \leqslant 1$ is a nondimensional parameter) is defined such that $P_{\delta W}\left(f_{t}^{c} d \Delta_{t}^{c}\right) \leqslant \gamma$ for all contacts $c \in C_{\delta W}(\gamma)$. Here $P_{\delta W}\left(f_{t} d \Delta_{t}\right)$ is the cumulative probability density function for the absolute values of the work increment $f_{t}^{c} d \Delta_{t}^{c}$ of the tangential mode. Thus if $c \in C_{\delta W}(\gamma)$ its tangential work increment is smaller than the $\gamma$ quantile. The "active" network consists of the contacts in the complement of $C_{\delta W}(\gamma)$, i.e., $C-C_{\delta W}(\gamma)$. The contacts that are part of the active network are called active, while the contacts that are not part of the active network are called inactive.

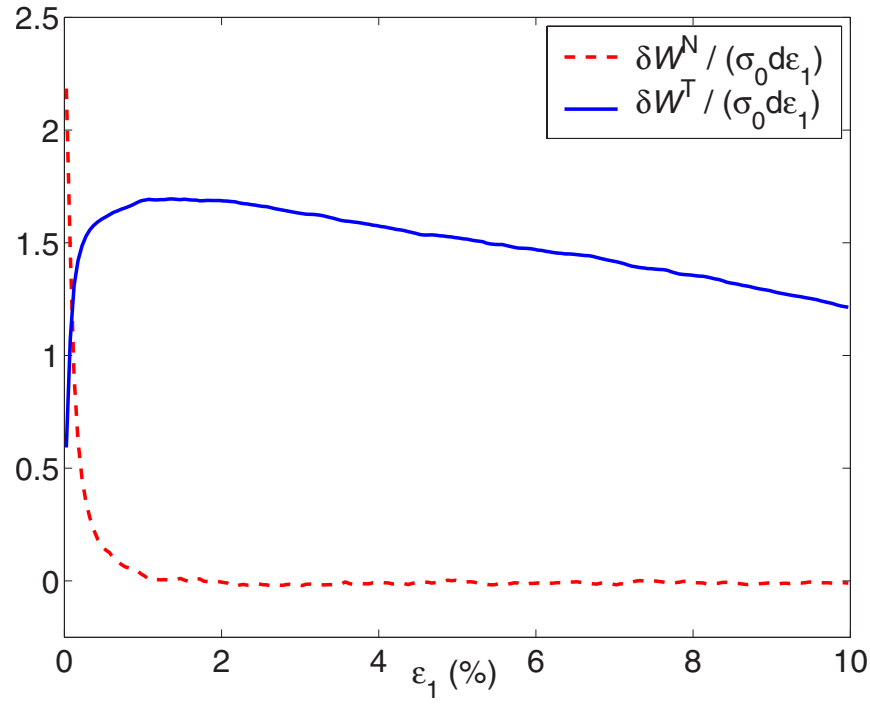

FIG. 6. (Color online) Evolution with imposed strain $\epsilon_{1}$ of contributions to the work increments of the normal and tangential modes, $\delta W^{N}$ and $\delta W^{T}$, respectively; $\sigma_{0}$ is the initial isotropic stress.

In analogy to the definitions of the partial stress and strain tensors in Eq. (5), the partial work increment $\delta W(\gamma)$ is defined as the work increment corresponding to the set $C_{\delta W}(\gamma)$,

$$
\delta W(\gamma)=\frac{1}{S_{c \in C_{\delta W(\gamma)}}} \sum_{n}\left[f_{n}^{c} d \Delta_{n}^{c}+f_{t}^{c} d \Delta_{t}^{c}\right] .
$$

Note that $\delta W(\gamma=1)=\delta W$.

The partial work increment $\delta W(\gamma)$ is shown in Fig. 7 at peak shear strength for various values of $\gamma$. The behavior at large strain is similar. We observe from Fig. 7 that at peak shear strength for $\gamma=0.5, \delta W(\gamma) / \delta W=0.04$ and for $\gamma=0.9$, $\delta W(\gamma) / \delta W=0.39$. This means that the $50 \%$ of the contacts at which the tangential work increment is smaller than the median tangential work increment contribute only $4 \%$ to the work increment. Similarly, the most active $10 \%$ of the con-

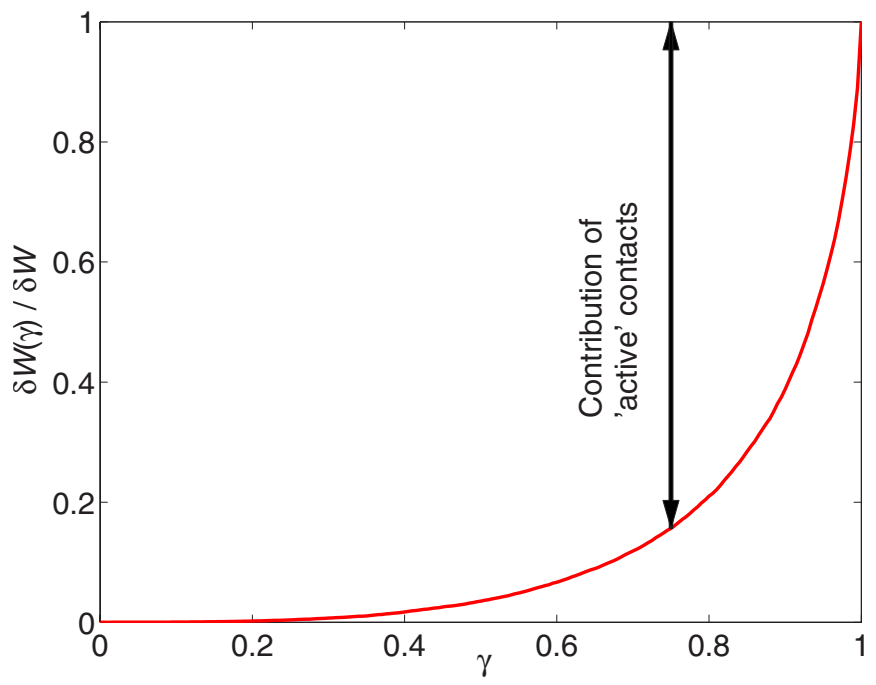

FIG. 7. (Color online) Partial work increment at peak shear strength. 
TABLE III. Percentages of various groups of contacts at peak shear strength: active vs strong and active vs mobile networks; $\alpha=\beta=\gamma=0.9$.

\begin{tabular}{lcccc}
\hline \hline & \multicolumn{4}{c}{ Percent of contacts } \\
\cline { 2 - 5 } $\begin{array}{l}\text { Type of } \\
\text { contact }\end{array}$ & Strong & Weak & Mobile & Immobile \\
\hline Active & 2.8 & 7.2 & 4.2 & 5.8 \\
Inactive & 7.2 & 82.8 & 5.8 & 84.2 \\
\hline \hline
\end{tabular}

tacts contribute $71 \%$ to the total work increment. Thus the active contacts determine the total work increment.

The relation of the active network with the strong network is investigated by considering the percentage of contacts in the four possible groups: active and strong, active and weak, inactive and strong, and inactive and weak. Similarly, the relation of the active network with the mobile network is investigated by considering the percentage of contacts in the four possible groups: active and mobile, active and immobile, inactive and mobile, and inactive and immobile.

For the specific choice of $\alpha=\beta=\gamma=0.9$, i.e., $10 \%$ strong and $10 \%$ mobile contacts and $10 \%$ active contacts, these percentages are given in Table III at peak shear strength. Note that since $\alpha=0.9$, the total percentage of strong contacts equals $10 \%$ and the total percentage of weak contacts equals $90 \%$. Similarly, since $\beta=0.9$, the total percentage of mobile contacts equals $10 \%$ and the total percentage of immobile contacts equals $90 \%$. Since $\gamma=0.9$, the total percentage of active contacts equals $10 \%$ and the total percentage of inactive contacts equals $90 \%$. If the active and strong networks were unrelated, then the percentage of active and strong contacts would equal $0.1 \times 0.1=1 \%$. Instead, this percentage equals $2.8 \%$. If the active and mobile networks were unrelated (or independent), then the percentage of active and mobile contacts would equal $1 \%$. Instead, this percentage equals $4.2 \%$. It appears that the active and the strong network are related. The same applies to a stronger degree to the active and mobile networks.

\section{DISCUSSION}

The contributions to the mean pressure and the shear stress of the normal and tangential forces have been analyzed. It has been shown that the contributions of the normal forces dominate over those due to the tangential forces. Similarly, the contributions to the volumetric and shear strain increments of the normal and tangential relative displacements have been analyzed. It has been demonstrated that the contributions of the tangential relative displacements domi- nate over those due to the normal relative displacements.

Following Radjaii [6], the strong network is identified at whose contacts the normal force is large. The strong network determines, to a large extent, the shear stress. In this study the dual viewpoint is considered by considering the relative displacements at contacts. It has been shown that a mobile network can be identified at whose contacts the tangential relative-displacement increment is large. This mobile network determines, to a large extent, the volumetric and shear strain increments.

For a proper description of quasistatic deformation of granular materials, the existence of strong and the mobile network must both be taken into account, since forces and relative displacements at contacts are related by the contact constitutive relation. It has been investigated whether the strong and the mobile network are related. The conclusion is that they are largely independent.

Since the stress is mainly determined by the normal forces, while the strain increment is mainly determined by the tangential relative displacements, the contributions to the work increment of the normal and tangential modes have been determined. It is found that the tangential mode dominates. An alternative network, based on a distinction between active and inactive contacts has been proposed.

These strong, mobile, and active networks have been considered here, since they are directly associated with macroscopic characteristics (stress and strain increment), contrary to other networks, such as a network based on contacts where slip occurs (fully mobilized friction at the contact).

A topic for further research is a comparison of the heterogeneous deformation of the mobile network with the deformation according to the recently introduced "spot model" $[40,41]$.

For micromechanical studies of constitutive relations for quasistatic deformation of granular materials, a distinction between a strong and a weak network, as suggested in [6], must accompany a distinction between the mobile and immobile network, as suggested here. It is an open issue whether the added complexity of constitutive closures for each of these networks forms a promising approach to the micromechanical modeling of quasistatic granular materials. This distinction does provide a clearer insight into the complex behavior of granular materials.

\section{ACKNOWLEDGMENTS}

The authors would like to acknowledge partial support for this study by the British Council and by NWO (Netherlands Foundation for Scientific Research) within the "UK-Netherlands Partnership Programme in Science."
[1] P. A. Cundall and O. D. L. Strack, Geotechnique 9, 47 (1979).

[2] A. Drescher and G. de Josselin de Jong, J. Mech. Phys. Solids 20, 337 (1972).

[3] M. Oda and J. Konishi, Soils Found. 14, 25 (1974).
[4] H. G. B. Allersma, J. Powder Bulk Solids Technol. 9, 7 (1985).

[5] T. S. Majmudar and R. P. Behringer, Nature (London) 435, 1079 (2005). 
[6] F. Radjaï, D. E. Wolf, M. Jean, and J. J. Moreau, Phys. Rev. Lett. 80, 61 (1998).

[7] J. F. Peters, M. Muthuswamy, J. Wibowo, and A. Tordesillas, Phys. Rev. E 72, 041307 (2005).

[8] M. E. Cates, J. P. Wittmer, J. P. Bouchaud, and P. Claudin, Phys. Rev. Lett. 81, 1841 (1998).

[9] M. E. Cates, J. P. Wittmer, J. P. Bouchaud, and P. Claudin, Chaos 9, 511 (1999).

[10] M. R. Kuhn, Mech. Mater. 31, 407 (1999).

[11] M. R. Kuhn, Granular Matter 4, 155 (2003).

[12] F. Alonso-Marroquin, I. Vardoulakis, H. J. Herrmann, D. Weatherley, and P. Mora, Phys. Rev. E 74, 031306 (2006).

[13] L. Rothenburg and A. P. S. Selvadurai, Proceedings International Symposium on Mechanical Behaviour of Structured Media, Ottawa, Canada, edited by A. P. S. Selvadurai (Elsevier, Amsterdam, 1981), pp. 469-486.

[14] N. P. Kruyt and L. Rothenburg, J. Appl. Mech. 63, 706 (1996).

[15] N. P. Kruyt, Int. J. Solids Struct. 40, 511 (2003).

[16] J. P. Bardet and I. Vardoulakis, Int. J. Solids Struct. 3, 353 (2001).

[17] K. Bagi, Int. J. Solids Struct. 40, 1329 (2003).

[18] M. R. Kuhn, Int. J. Solids Struct. 40, 1805 (2003).

[19] W. Ehlers, E. Ramm, S. Diebels, and G. A. d'Addetta, Int. J. Solids Struct. 40, 6681 (2003).

[20] F. Froiio, G. Tomassetti, and I. Vardoulakis, Int. J. Solids Struct. 43, 7684 (2006).

[21] M. R. Kuhn and K. Bagi, Int. J. Solids Struct. 41, 5793 (2004).

[22] K. Bagi, Int. J. Solids Struct. 43, 3166 (2006).

[23] C. S. O'Hern, L. E. Silbert, A. J. Liu, and S. R. Nagel, Phys. Rev. E 68, 011306 (2003).

[24] M. Wyart, S. R. Nagel, and T. A. Witten, Europhys. Lett. 72, 486 (2005).
[25] L. E. Silbert, A. J. Liu, and S. R. Nagel, Phys. Rev. E 73, 041304 (2006).

[26] W. G. Ellenbroek, E. Somfai, M. van Hecke, and W. van Saarloos, Phys. Rev. Lett. 97, 258001 (2006).

[27] C. Thornton, Geotechnique 50, 43 (2000).

[28] C. Thornton and S. J. Antony, Philos. Trans. R. Soc. London, Ser. A 356, 2763 (1998).

[29] C. Thornton and D. J. Barnes, Acta Mech. 64, 45 (1986).

[30] L. Rothenburg and R. J. Bathurst, Geotechnique 39, 601 (1989).

[31] N. P. Kruyt, Int. J. Solids Struct. 40, 3537 (2003).

[32] N. P. Kruyt and L. Rothenburg, Proceedings Quasi-static Deformations of Particulate Materials, edited by K. Bagi (Budapest University of Technology and Economics, Budapest, Hungary, 2003), pp. 141-150.

[33] S. J. Antony and M. R. Kuhn, Int. J. Solids Struct. 41, 5863 (2004).

[34] F. Radjaï and S. Roux, Phys. Rev. Lett. 89, 064302 (2002).

[35] I. K. Ono, S. Tewari, S. A. Langer, and A. J. Liu, Phys. Rev. E 67, 061503 (2003).

[36] N. P. Kruyt and L. Rothenburg, Mech. Mater. 36, 1157 (2004).

[37] H. A. Makse, N. Gland, D. L. Johnson, and L. M. Schwartz, Phys. Rev. Lett. 83, 5070 (1999).

[38] L. Rothenburg and N. P. Kruyt, Powders \& Grains 2001: 4th International Conference on Micromechanics of Granular Media, edited by Y. Kishino (Balkema, Lisse, The Netherlands, 2001), pp. 191-194.

[39] N. P. Kruyt and L. Rothenburg, J. Stat. Mech.: Theory Exp. 2006, P07021.

[40] M. Z. Bazant, Mech. Mater. 38, 717 (2006).

[41] C. H. Rycroft, M. Z. Bazant, G. S. Grest, and J. W. Landry, Phys. Rev. E 73, 051306 (2006). 